\title{
Order in Quantum Compass and Orbital $e_{g}$ Models
}

\author{
P. Czarnik ${ }^{a, b}$, J. DZiarmaga ${ }^{a}$ And A.M. Oleś $\hat{S}^{a, c, *}$ \\ ${ }^{a}$ M. Smoluchowski Institute of Physics, Jagiellonian University, prof. S. Łojasiewicza 11, PL-30348 Kraków, Poland \\ ${ }^{b}$ Institute of Nuclear Physics, Polish Academy of Sciences, Radzikowskiego 152, PL-31342 Kraków, Poland \\ ${ }^{c}$ Max Planck Institute for Solid State Research, Heisenbergstrasse 1, D-70569 Stuttgart, Germany
}

\begin{abstract}
We investigate thermodynamic phase transitions in the compass model and in $e_{g}$ orbital model on an infinite square lattice by variational tensor network renormalization (VTNR) in imaginary time. The onset of nematic order in the quantum compass model is estimated at $\mathcal{T}_{c} / J=0.0606(4)$. For the $e_{g}$ orbital model one finds: (i) a very accurate estimate of $\mathcal{T}_{c} / J=0.3566 \pm 0.0001$ and $(i i)$ the critical exponents in the Ising universality class. Remarkably large difference in frustration results in so distinct values of $\mathcal{T}_{c}$, while entanglement influences the quality of $\mathcal{T}_{c}$ estimation.
\end{abstract}

DOI: 10.12693/APhysPolA.133.359

PACS/topics: 75.10.Jm, 05.10.Cc, 05.70.Fh, 75.25.Dk

\section{Introduction}

In a two-dimensional (2D) spin systems with exchange interactions having $\mathrm{SU}(2)$ symmetry long-range order is excluded as stated by Mermin and Wagner [1]. This paradigm fails in generic orbital models, such as the $2 \mathrm{D}$ compass [2] or 2D $e_{g}$ orbital [3] model, where interactions do not satisfy the assumptions of the MerminWagner theorem and the involved pseudospins order below a thermodynamic phase transition at finite temperature $\mathcal{T}_{c}$. Exchange interactions in both $e_{g}$ orbital and compass model may be derived from the Ising model when the anisotropy and frustration of exchange interactions between the two $a$ and $b$ axes in the square lattice increases [4]. Maximal frustration occurs in the 2D compass model and the nematic order is predicted by Quantum Monte Carlo (QMC) calculations below a rather low temperature $\mathcal{T}_{c}=0.0585 J$ [5]. The $e_{g}$ orbital model orders as well [6] but the value of $\mathcal{T}_{c}$ is unknown as QMC calculations fail due to the sign problem.

Orbital models arise in a natural way when intraorbital Coulomb interaction $U$ for partly filled $3 d$ orbitals is large compared with the hopping element $t$, electrons localize and the effective interactions for a strongly correlated transition metal oxide with orbital degeneracy are given by spin-orbital superexchange. Kugel and Khomskii [7] were the first to recognize that spins and orbitals are quantum and have to be treated on equal footing and their interplay may lead to spectacular symmetry broken phases. Since then various compounds are treated in detail and the field developed to spin-orbital physics [8-13]. Spin-orbital models relevant for real materials are quite involved and depend on the type of orbital degree of freedom, $e_{g}$ or $t_{2 g}$. While $e_{g}$ orbital interactions control the orbital order in ferromagnetic planes of $\mathrm{KCuF}_{3}$ and $\mathrm{LaMnO}_{3}$ which is quite robust and survives for spin

*corresponding author; e-mail: a.m.oles@fkf.mpg.de disorder [14, 15], compass interactions stand for the pseudospin exchange in systems with strong spin-orbit coupling at $4 d$ (or $5 d$ ) ions on a square lattice [16].

The purpose of this paper is to present the results obtained with Variational Tensor Network Renormalization (VTNR) in imaginary time [17]. This algorithm approximates thermal density matrix $\rho(\mathcal{T}) \propto e^{-H / \mathcal{T}}$ of a $2 \mathrm{D}$ quantum lattice model with a Hamiltonian $H$ by a pair entangled projected operator (PEPO) [18] which is an example of a tensor network [19]. The refinement parameter is bond dimension $D$. Small $D$ is sufficient to have exact $\rho(\mathcal{T})$ representation for a classical $H$ (e.g. $D=2$ for spins or pseudospins 1/2). Larger $D$ enables VTNR to capture effects of quantum fluctuations and entanglement [18]. In the limit $D \rightarrow \infty$ one can always recover non-truncated $\rho(\mathcal{T})$ although polynomial scaling of CPU time with $D$ restricts numerical calculations to $D \leqslant 20$ in practice. The method like all tensor network methods doesn't suffer from the sign problem which makes it powerful tool for simulation of weakly entangled models. VTNR was successfully applied to the 2D Hubbard model at $\mathcal{T}>0[20]$ and to estimate the value of $\mathcal{T}_{c}$ for both the 2D compass [21] and the $e_{g}$ orbital model [22].

We discuss the nematic order in the $2 \mathrm{D}$ compass model in Sect. 2. Next we present in Sect. 3 a very accurate estimate of $\mathcal{T}_{c}$ and the critical exponents $\beta$ and $\gamma$ for the $2 \mathrm{D} e_{g}$ orbital model. The paper is summarized in Sect. 4 with a comparison between frustrated Ising and two quantum models, $e_{g}$ orbital and compass.

\section{Nematic order in the compass model}

The quantum compass model on a square lattice is [2],

$$
H_{\text {com }}=-\frac{1}{4} J \sum_{j} X_{j} X_{j+e_{a}}-\frac{1}{4} J \sum_{j} Z_{j} Z_{j+e_{b}} .
$$

Here $j$ is a site index, $X_{j} \equiv \sigma_{j}^{x}$ and $Z_{j} \equiv \sigma_{j}^{z}$ are Pauli matrices at site $j$, and $e_{a}\left(e_{b}\right)$ are unit vectors along the $a(b)$ axis. As in the Ising model the sign of $J$ in equation (1) may be arbitrary and we take ferromagnetic exchange $J=1:-\frac{1}{4} X_{j} X_{j+e_{a}}\left(-\frac{1}{4} Z_{j} Z_{j+e_{a}}\right)$ for a bond along the 
$a(b)$ axis. The order parameter $Q>0$ is,

$$
Q \equiv\left|\left\langle Q_{j}\right\rangle_{\mathcal{T}}\right|=\left|\left\langle X_{j} X_{j+e_{a}}-Z_{j} Z_{j+e_{b}}\right\rangle_{\mathcal{T}}\right|
$$

This order parameter is finite below the phase transition at $\mathcal{T}_{c}$ - the transition belongs to the 2D Ising universality class [23]. As expected for any $\mathcal{T}>0$ we find that local order parameters vanish within the numerical precision of $10^{-5}$, i.e., $\left\langle X_{j}\right\rangle_{\mathcal{T}}=\left\langle Z_{j}\right\rangle_{\mathcal{T}}=0$. Strong frustration implies that there is neither any local magnetization nor any long-range order.

For the phase transition to nematic order in the $2 \mathrm{D}$ compass model a value of $\mathcal{T}_{c}=0.0585$ was estimated by QMC [23], while a high-temperature series expansion predicts a similar value $\mathcal{T}_{c}=0.0625$ [24]. The value of $\mathcal{T}_{c}$ is strongly suppressed by quantum fluctuations from the classical compass model [5]. The high-temperature extrapolation gives the susceptibility exponent $\gamma \simeq 1.3$. Our estimate $\gamma=1.35$ is in the same range and the order parameter $Q(\mathcal{T})$ exponent is $\beta=0.223$ [21]. Unfortunately the convergence in $D$ is rather slow [21], so these values are close but not sufficiently close to the exact $2 \mathrm{D}$ Ising values, $\gamma=\frac{7}{4}$ and $\beta=\frac{1}{8}$.

The nature of nematic order in the $2 \mathrm{D}$ quantum compass model is better understood by studying its symmetries. The spectral properties can be uniquely determined by discrete symmetries like parity. The conservation of spin parities in rows and columns in the $2 \mathrm{D}$ quantum compass model (for $X$ and $Z$ operators) uncovers very interesting hidden order with two-dimer correlations [25]. The two-site correlations (2) in the nematic phase are shown in Fig. 1. The dominant correlation function (here

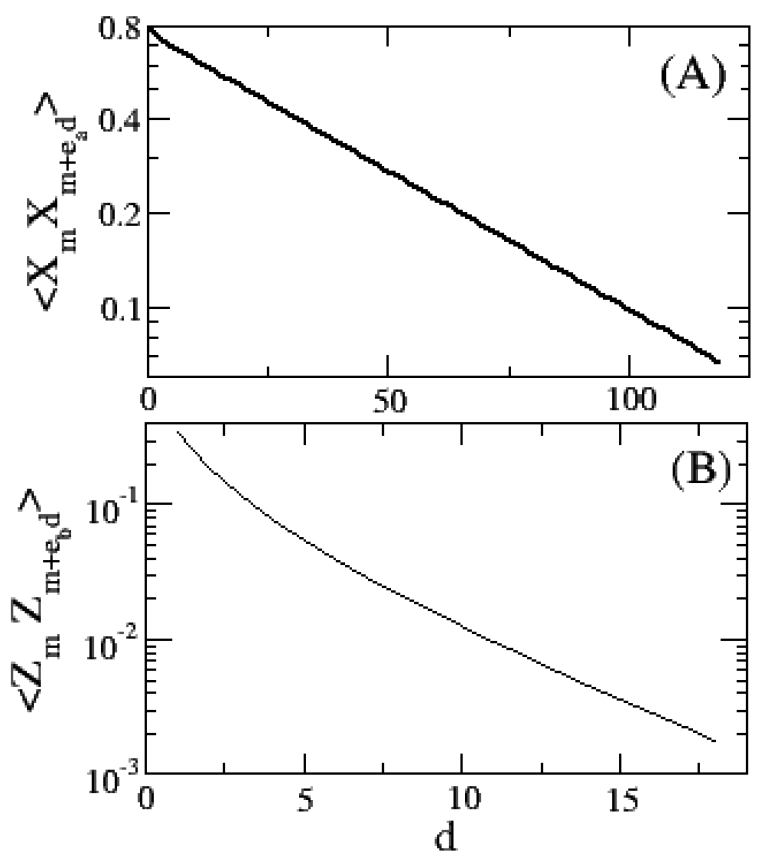

Fig. 1. Nematic order in the compass model at $\mathcal{T}=0.05814$ : Spin correlations for increasing distance $d$ along the $a(b)$ axis: (A) the dominant correlation $\left\langle X_{m} X_{m+e_{a} d}\right\rangle$ [21], and (B) the transverse correlation $\left\langle Z_{m} Z_{m+e_{b} d}\right\rangle$, both for $D=15$. along the $a$ axis) is exponential but relatively long-ranged with a correlation length estimated at $\xi_{\|}=40(2)$ [21]. The transverse correlations are weaker and decay nearly exponentially with a much shorter length $\xi_{\perp}=6.9(4)$.

\section{Ising-like order in the $e_{g}$ model}

The quantum $e_{g}$ model on an infinite square lattice is obtained by a proper transformation from the 2D Ising model [4] and is defined by the Hamiltonian,

$$
H_{3}=-J \sum_{j} \tau_{j}^{a} \tau_{j+e_{a}}^{a}-J \sum_{j} \tau_{j}^{b} \tau_{j+e_{b}}^{b} .
$$

The notation is analogous as in (1) with the coupling of different orbital operators along the $a$ and $b$ axis:

$$
\tau_{j}^{a}=\frac{1}{4}\left(-\sigma_{j}^{z}+\sqrt{3} \sigma_{j}^{x}\right), \tau_{j}^{b}=\frac{1}{4}\left(-\sigma_{j}^{z}-\sqrt{3} \sigma_{j}^{x}\right) .
$$

As in Sect. 2, we take the isotropic model with $J=1$.

At low temperature a spontaneous breaking of symmetry is determined by the term $\propto \frac{3}{16} \sigma_{i}^{x} \sigma_{j}^{x}$ [4]. This symmetry breaking implies a finite order parameter

$$
m(\mathcal{T}) \equiv\left\langle\sigma_{j}^{x}\right\rangle_{\mathcal{T}}
$$

It could be obtained by a systematic convergence test in the bond dimension $D$. Indeed, from $D=4$ the data practically fall on each other and $D=8$ corresponds to the converged results, see Fig. 2. The entanglement at $\mathcal{T} \rightarrow 0$ is small and therefore $m(\mathcal{T})$ converges in $D$ so fast. The ground state order parameter is almost saturated, $m(0)=0.993$, in agreement with the Multiscale Entanglement Renormalization Ansatz (MERA) [4], and the quantum fluctuations are very weak at $\mathcal{T}=0$ [3].

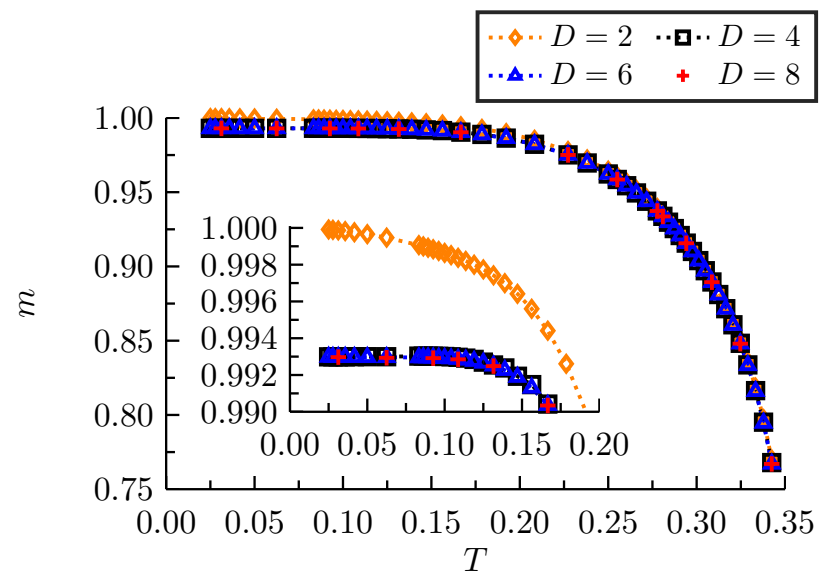

Fig. 2. The order parameter $m(\mathcal{T})(5)$ as a function of temperature $\mathcal{T}$ below the critical regime. The inset shows the zoom of $m(\mathcal{T})$ at low temperature $\mathcal{T}<0.18$. The results demonstrate fast convergence in $D-$ only $D=2$ gives a higher $m(\mathcal{T})$, while the data for $D=$ $4,6,8$ all overlap.

Unlike the 2D compass model [23], the $e_{g}$ orbital model (2) is not tractable by QMC [26], but the order parameter $m(\mathcal{T})$ and the susceptibility $\chi(\mathcal{T})$ were found by the VTNR Anzatz. Their behavior near $\mathcal{T}_{c}$ is:

$$
m(\mathcal{T}) \propto\left(\mathcal{T}_{c}-\mathcal{T}\right)^{\beta}, \quad \chi(\mathcal{T}) \propto\left(\mathcal{T}-\mathcal{T}_{c}\right)^{\gamma} .
$$

The convergence in $D$ is fast [22] and one finds: $\beta \simeq 0.126$ 
TABLE I

The critical temperature $\mathcal{T}_{c}$ and the type of order for the classical and quantum models on a square lattice: Ising model, $\frac{1}{2}$ and $\frac{2}{3}$ frustrated Ising [29], fully frustrated Villain model [30], $e_{g}$ orbital model [22] and 2D compass model [21].

\begin{tabular}{c|c|c|c|c}
\hline \hline 2D model & order & $\mathcal{T}_{c} / J$ & method & Ref. \\
\hline Ising & $2 \mathrm{D}$ & 0.567296 & exact & {$[27]$} \\
$\frac{1}{2}$ frustrated & $2 \mathrm{D}$ & 0.410 & exact & {$[29]$} \\
$\frac{2}{3}$ frustrated & $2 \mathrm{D}$ & 0.342 & exact & {$[29]$} \\
Villain & - & 0.0 & exact & {$[30]$} \\
\hline$e_{g}$ orbital & 2D & $0.3566 \pm 0.0001$ & VTNR & {$[22]$} \\
compass & nematic & $0.0606 \pm 0.0004$ & VTNR & {$[21]$}
\end{tabular}

and $\gamma \simeq 1.736$ for $D \geqslant 7$; they approach the critical exponents of the 2D Ising model, $\beta=\frac{1}{8}$ and $\gamma=\frac{7}{4}$. From the convergence of $m(\mathcal{T})$ and $\chi(\mathcal{T})$ (6) we deduce $\mathcal{T}_{c} \simeq 0.3566[22]$. Note that from the leading $\propto \frac{3}{16} \sigma_{i}^{x} \sigma_{j}^{x}$ term in (2) and the Ising model [27] one expects $\mathcal{T}_{c} \simeq 0.4255$ but the quantum fluctuations are activated by temperature and reduce the above value of $\mathcal{T}_{c}$ by $\sim 16 \%$.

\section{Summary}

To highlight the difference between geometrical frustration [28] in classical and intrinsic frustration [12] in quantum models, we compare the values of $\mathcal{T}_{c}$ for the $2 \mathrm{D}$ $e_{g}$ orbital and compass models with those for 2D frustrated Ising model in Table I. Increasing the number of frustrated plaquettes on a square lattice reduces $\mathcal{T}_{c}$ but as long as ladders of non-frustrated plaquettes exist, the decrease of $\mathcal{T}_{c}$ is slow [29], and only when all the plaquettes are frustrated the $2 \mathrm{D}$ order totally collapses in the Villain model [30]. In contrast, for complete quantum frustration in the compass model, the ground state is highly degenerate but a novel nematic order emerges below $\mathcal{T}_{c}>0$. For partial frustration in the $e_{g}$ orbital model the reduction of $\mathcal{T}_{c}$ is more dramatic as quantum fluctuations are activated in the critical regime.

To summarize, by comparing the $e_{g}$ orbital and quantum compass model we conclude that the VTNR Ansatz is particularly efficient when entanglement is weak. Then a very accurate treatment is possible, even when the system suffers from the fermionic sign problem. We suggest that both the $2 \mathrm{D} e_{g}$ and compass model are in the $2 \mathrm{D}$ Ising universality class and present a very accurate estimate of $\mathcal{T}_{c}$ for the less entangled $e_{g}$ model.

\section{Acknowledgments}

We kindly acknowledge support by Narodowe Centrum Nauki (NCN) under Projects No. 2016/23/B/ST3/00830 (P.C. and J.D.), No. 2016/23/B/ST3/00839 (A.M.O.), and No. 2015/16/T/ST3/00502 (Ph.D. thesis of P.C.).

\section{References}

[1] N.D. Mermin, H. Wagner, Phys. Rev. Lett. 17, 1133 (1966).

[2] Z. Nussinov, J. van den Brink, Rev. Mod. Phys. 86, $1(2015)$
[3] J. van den Brink, P. Horsch, F. Mack, A.M. Oleś, Phys. Rev. B 59, 6795 (1999).

[4] L. Cincio, J. Dziarmaga, A.M. Oleś, Phys. Rev. B 82, 104416 (2010).

[5] S. Wenzel, W. Janke, Phys. Rev. B 78, 064402 (2008).

[6] A. van Rynbach, S. Todo, S. Trebst, Phys. Rev. Lett. 105, 146402 (2010).

[7] K.I. Kugel, D.I. Khomskii, Sov. Phys. Usp. 25, 231 (1982).

[8] Y. Tokura, N. Nagaosa, Science 288, 462 (2000).

[9] A.M. Oleś, G. Khaliullin, P. Horsch, L.F. Feiner, Phys. Rev. B 72, 214431 (2005).

[10] G. Khaliullin, Prog. Theor. Phys. Suppl. 160, 155 (2005).

[11] B. Normand, A.M. Oleś, Phys. Rev. B 78, 094427 (2008); B. Normand, Phys. Rev. B 83, 064413 (2011); J. Chaloupka, A.M. Oleś, Phys. Rev. B 83 094406 (2011).

[12] A.M. Oleś, J. Phys.: Condens. Mat. 24, 313201 (2012); Acta Phys. Pol. A 127, 163 (2015).

[13] W. Brzezicki, A.M. Oleś, M. Cuoco, Phys. Rev. X 5, 011037 (2015); W. Brzezicki, M. Cuoco, A.M. Oleś, J. Sup. Novel Magn. 29, 563 (2016); ibid. 30, 129 (2017); W. Brzezicki, M. Cuoco, F. Forte, A.M. Oleś, ibid. 31, 639 (2018).

[14] A.J.W. Reitsma, L.F. Feiner, A.M. Oleś, New J. Phys. 7, 121 (2005).

[15] M. Snamina, A.M. Oleś, Phys. Rev. B 94, 214426 (2016).

[16] G. Jackeli, G. Khaliullin, Phys. Rev. Lett. 102 , 017205 (2009).

[17] P. Czarnik, J. Dziarmaga, Phys. Rev. B 92, 035120 (2015);ibid. 92, 035152 (2015).

[18] M.M. Wolf, F. Verstraete, M.B. Hastings, J.I. Cirac, Phys. Rev. Lett. 100, 070502.

[19] U. Schollwöck, Ann. of Phys. 326, 96 (2011).

[20] P. Czarnik, M.M. Rams, J. Dziarmaga, Phys. Rev. B 94, 235142 (2016).

[21] P. Czarnik, J. Dziarmaga, A.M. Oleś, Phys. Rev. B 93, 184410 (2016).

[22] P. Czarnik, J. Dziarmaga, A.M. Oleś, Phys. Rev. B 96, 014420 (2017).

[23] S. Wenzel, W. Janke, A.M. Läuchli, Phys. Rev. E 81, 066702 (2010)

[24] J. Oitmaa, C.J. Hamer, Phys. Rev. B 83, 094437 (2011).

[25] W. Brzezicki, A.M. Oleś, Phys. Rev. B 82, 060401 (2010); ibid. 87, 214421 (2013).

[26] S. Wenzel, A.M. Läuchli, Phys. Rev. Lett. 106 197201 (2011).

[27] L. Onsager, Phys. Rev. 65, 117 (1944).

[28] D.C. Mattis, The Theory of Magnetism Made Simple, World Scientific, New Jersey, 2006.

[29] L. Longa, A.M. Oleś, J. Phys. A: Math. Theor. 13, 1031 (1980)

[30] J. Villain, J. Phys. C: Solid State Phys. 10, 1717 (1977). 